
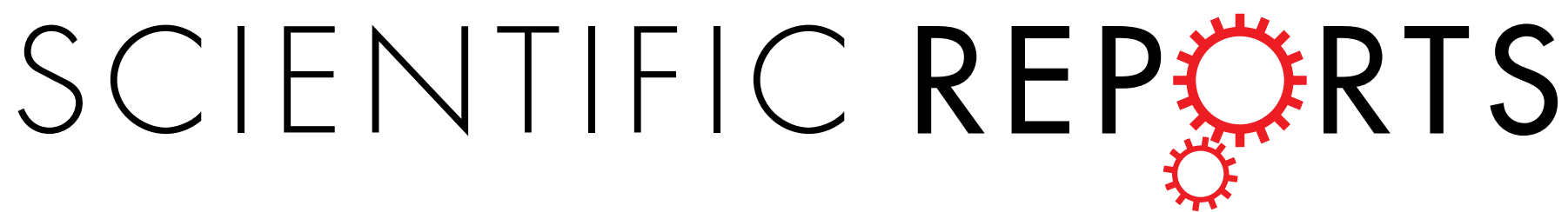

Mycobacteriophage SWU1 gp39 can potentiate multiple antibiotics against Mycobacterium via altering

Received: 23 March 2016

Accepted: 08 June 2016

Published: 28 June 2016

\section{the cell wall permeability}

Qiming $\mathrm{Li}^{1, *}$, Mingliang Zhou ${ }^{1, *}$, Xiangyu Fan ${ }^{1,2, *}{ }^{*}$, Jianlong $\mathrm{Yan}^{1}{ }^{1}$, Weimin $\mathrm{Li}^{3}$ \& Jianping $\mathrm{Xie}^{1}$

M. tuberculosis is intrinsically tolerant to many antibiotics largely due to the imperviousness of its unusual mycolic acid-containing cell wall to most antimicrobials. The emergence and increasingly widespread of multidrug-resistant tuberculosis (MDR-TB) and extensively drug-resistant tuberculosis (XDR-TB) revitalized keen interest in phage-inspired therapy. SWU1gp39 is a novel gene from mycobacteriophage SWU1 with unknown function. SWU1gp39 expressed in M. smegmatis conferred the host cell increased susceptibility to multiple antibiotics, including isoniazid, erythromycin, norfloxacin, ampicillin, ciprofloxacin, ofloxacin, rifampicin and vancomycin, and multiple environment stresses such as $\mathrm{H}_{2} \mathrm{O}_{2}$, heat shock, low pH and SDS. By using EtBr/Nile red uptake assays, WT-pAL-gp39 strain showed higher cell wall permeability than control strain WT-pAL. Moreover, the WT-pAL-gp39 strain produced more reactive oxygen species and reduced NAD ${ }^{+} / N A D H$ ratio. RNA-Seq transcriptomes of the WT-pAL-gp39 and WT-pAL revealed that the transcription of 867 genes was differentially regulated, including genes associated with lipid metabolism. Taken together, our results implicated that SWU1gp39, a novel gene from mycobacteriophage, disrupted the lipid metabolism of host and increased cell wall permeability, ultimately potentiated the efficacy of multiple antibiotics and stresses against mycobacteria.

Tuberculosis, caused by Mycobacterium tuberculosis (M. tuberculosis), remains a major global public health concern. The emergence of multidrug-resistant $M$. tuberculosis (MDR-MTB) and extensively drug-resistant M. tuberculosis (XDR-MTB) strains exacerbated the situation. M. tuberculosis is intrinsically tolerant to many antibiotics largely due to the imperviousness of its unusual mycolic acid-containing cell wall to most chemotherapeutics and inducible expression of several active drug efflux pumps. Some genes involved in metabolism or other processes were identified to mediate intrinsic resistance in mycobacteria. The inactivation of asparagine synthetase AsnB, an asparagine biosynthetic enzyme catalyzing the transfer of the $\gamma$-amino residue of glutamine to the carboxyl residue of aspartate, dramatically sensitized Mycobacterium smegmatis (M. smegmatis) to multiple antibiotics, including rifampin, erythromycin, novobiocin and fusidic acid ${ }^{1}$. Mycobacteria proteasome accessory facror ( $p a f C$ ), a component of bacterial proteasome, was identified to be involved in fluoroquinolones intrinsic resistance. PafC inactivation mutants are specifically hypersensitive to fluoroquinolones, including moxifloxacin, norfloxacin, ofloxacin, ciprofloxacin, but not to other antibiotics such as isoniazid, rifampicin, spectinomycin, chloramphenicol, capreomycin ${ }^{2}$. Protein kinase G (pknG), Serine/threonine protein kinase, was also required for intrinsic antibiotic resistance in mycobacteria ${ }^{3}$. In M. smegmatis, another Serine/threonine protein kinase MSMEG_5437 and a predicated anti-sigma factor MSMEG_6129 were involved in intrinsic resistance to a variety of stresses including the genotoxic agent mitomycin $\mathrm{C}$, hydrogen peroxide and at least four different

${ }^{1}$ Institute of Modern Biopharmaceuticals, State Key Laboratory Breeding Base of Eco-Environment and BioResource of the Three Gorges Area, Key Laboratory of Eco-environments in Three Gorges Reservoir Region, Ministry of Education, School of Life Sciences, Southwest University, Beibei, Chongqing 400715, China. ${ }^{2}$ School of Biological Science and Technology, University of Jinan, Shandong 250022, China. ${ }^{3}$ National Tuberculosis Clinical Lab of China, Beijing Key laboratory on Drug-resistant Tuberculosis Research, Beijing Tuberculosis and Thoracic Tumor Research Institute, Beijing Chest Hospital, Capital Medical University, Beijing 101149, China. *These authors contributed equally to this work. Correspondence and requests for materials should be addressed to W.L. (email: Iwm_18@aliyun.com) or J.X. (email: georgex@swu.edu.cn) 
antibiotics, namely isoniazid, chloramphenicol, erythromycin and tetracycline ${ }^{4}$. Isocitrate lyases (ICLs), metabolic enzymes previously recognized as dedicated to the replenishing of tricarboxylic acid (TCA) cycle intermediates, was recently shown to have unexpected role in antioxidant defense as a mechanism of antibiotic tolerance ${ }^{5}$. Cytochrome $b d$ was found to play a key role in the survival of mycobacteria during multiple lethal stress, including hydrogen peroxide, clofazimine and bedaquiline $e^{6,7}$. The intrinsic drug resistance of mycobacteria represents formidable obstacle to tuberculosis control. It is imperative to find novel therapeutic or synergetic targets that can potentiate the antimicrobial lethality against tuberculosis.

Despite the highly impermeable mycolic acid containing cell wall, several first-line or second-line anti-tuberculosis drugs targeting cell wall are in clinical use, such as Isoniazid (INH) which can disrupt the cell wall integrity by inhibiting mycolic acid biosynthesis ${ }^{8}$. Upon entry into M.tuberculosis, the prodrug INH is activated to form an isonicotinyl-free radical by the bacterial peroxidase/catalase kat $\mathrm{G}^{9}$. The $\mathrm{INH}$-free radical reacts with NAD to form an INH-NAD complex that is a highly potent inhibitor of the essential enzyme InhA, an enoyl-ACP reductase required for mycolic acid biosynthesis ${ }^{10,11}$. Multiple factors contributing to clinical isolates INH resistance limited the efficacy of $\mathrm{INH}^{12,13}$. Ethionamide, a structural analog of INH, is another anti-TB drug that inhibits InhA activity by a mechanism of action similar to that of INH but requires activation by EthA instead of $\mathrm{katG}^{10}$. Mutations in the inhA structural gene or promoter region underpin the resistance to ETH. Other factors such as alterations in the drugs activators expression, redox status change, drug inactivation, and drug efflux pump activation ${ }^{14}$, contribute to the resistance to INH and ETH. New anti-TB drugs targeting cell wall are needed to combat tuberculosis.

Bacteriophage, the virus of bacteria, is a useful tool to control bacteria infections. Endolysins are peptidoglycan hydrolases encoded by bacteriophages that can break down bacterial peptidoglycan at the terminal stage of the phage reproduction cycle ${ }^{15}$. Endolysins are in trial as potential antimicrobial agents, especially for multidrug-resistant bacteria. Abp1, a virulent phage targeting the multidrug-resistant Acinetobacter baumannii, encoding an endolysin PlyAB1 exhibited significant antibacterial activity against all 48 clinical PDRAB isolates within a relatively short time frame ${ }^{16}$. Many mycobacteriophage-derived endolysins have shown antimycobacterial activity, including mycobacteriophage Bxz $2^{17}, \mathrm{BTCU}-1^{18}, \mathrm{Ms} 6^{19,20}, \mathrm{D} 29^{21}$. The lytic endolysins are intensively pursued as alternative sanitation or disinfectant agents against mycobacterium infections. With an estimated huge biomass of $10^{31}$ phages worldwide, phage-encoded endolysins and other effective proteins represent vast reservoir for novel antimicrobials. Bacteriophage P1-encoded Ref protein, a novel class of endonuclease, is toxic specifically during the bacterial SOS response or the stationary phase cultures ${ }^{22}$. These studies implicate that phage-based therapy can be alternative or complement to antibiotics.

In this study, we identified a novel gene gp39 from mycobacteriophage SWU1, which was absent in mycobacteriophage L5, although SWU1 and L5 are highly similar. We expressed SWU1gp39 in M. smegmatis using vector $p A L A C E$ under the control of an acetamide inducible promoter. The recombinant strain (WT-pAL-gp39, induced by acetamide) showed growth deficiency in medium containing hygromycin in comparison with the control strain (WT-pAL), although $p A L A C E$ contains a hygromycin resistance cassette. SWU1gp39 expressed in $M$. smegmatis leads to increased susceptibility to multiple antibiotics, including isoniazid, erythromycin, norfloxacin, ampicillin, ciprofloxacin, ofloxacin, rifampicin and vancomycin, also some environmental stresses such as $\mathrm{H}_{2} \mathrm{O}_{2}$, SDS, low $\mathrm{pH}$ and heat shock. Using RNA-seq, we found that the regulatory and metabolic pathways were changed by SWU1gp39. Scanning electron microscopy (SEM) and ethidium bromide or nile red uptake assays showed the morphology and cell wall permeability alteration in the recombinant strain.

\section{Results}

Gp39 is a novel gene from Mycobacteriophage SWU1. Mycobacteriophage SWU1 is a newly isolated phage from soil sample collected in Sichuan province, China ${ }^{23}$, which is highly similar to mycobacteriophage $\mathrm{L}^{24}$, but no homolog of SWU1gp39 was found in the L5 genome (Fig. 1A). No homolog was found in NCBI using BLAST until mycobacteriophage EagleEye and Serenity were isolated (Fig. 1B). To define the function of SWU1gp39, it was amplified from SWU1 genome (Fig. 1C) and expressed a His-tagged gp39 protein using a recombinant pALACE plasmid (Fig. 1D).

SWU1gp39 expression affects host growth upon exposure to several antibiotics. To test the effect of SWU1gp39 on recombinant strains, the growth rates of WT-pAL and WT-pAL-gp39 were monitored at $\mathrm{OD}_{600}$ after initial inoculation at the same concentration. As shown in Fig. 2, no growth differences were detected between WT-pAL and WT-pAL-gp39, demonstrating that the expression of SWU1gp39 does not affect the growth of $M$. smegmatis in $7 \mathrm{H} 9$ medium supplemented with $0.05 \%$ Tween 80 and $0.2 \%$ glycerinum. In the presence of Hyg, WT-pAL cells started to grow and entered into logarithmic phase $3 \mathrm{~h}$ delay in comparison to the absence of Hyg. In contrast, WT-pAL-gp39 treated with Hyg was incapable of growth though pALACE containing a Hyg resistance cassette (Fig. 2A). Similar growth defect was found when WT-pAL and WT-pAL-gp39 grown in M9 medium supplemented with glucose as the only carbon source, however WT-pAL-gp39 strain can grow to logarithmic phase after $60 \mathrm{~h}$ (Fig. 2B). To determine whether the homologous genes from EagleEye and Serenity possess similar function, the related genes were synthesized by commercial company and expressed in M. smegmatis by using the same shuttle plasmid pALACE (named WT-pAL-E and WT-pAL-S) (Fig. 1E). As expected, WT-pAL-E and WT- pAL-S also showed some growth defect in the presence of Hyg (Fig. 2C,D). The growth rates were also tested when WT-pAL and WT-pAL-gp39 exposed to other antibiotics on the plates. As shown in Fig. 2E, prominent growth defect was observed upon exposure to isoniazid, erythromycin, norfloxacin and ampicillin. Though M. smegmatis possess intrinsic resistance to amplicillin due to its cell wall complex structure, overexpressed SWU1gp39 can potentiate the antibiotic sensitivity. 

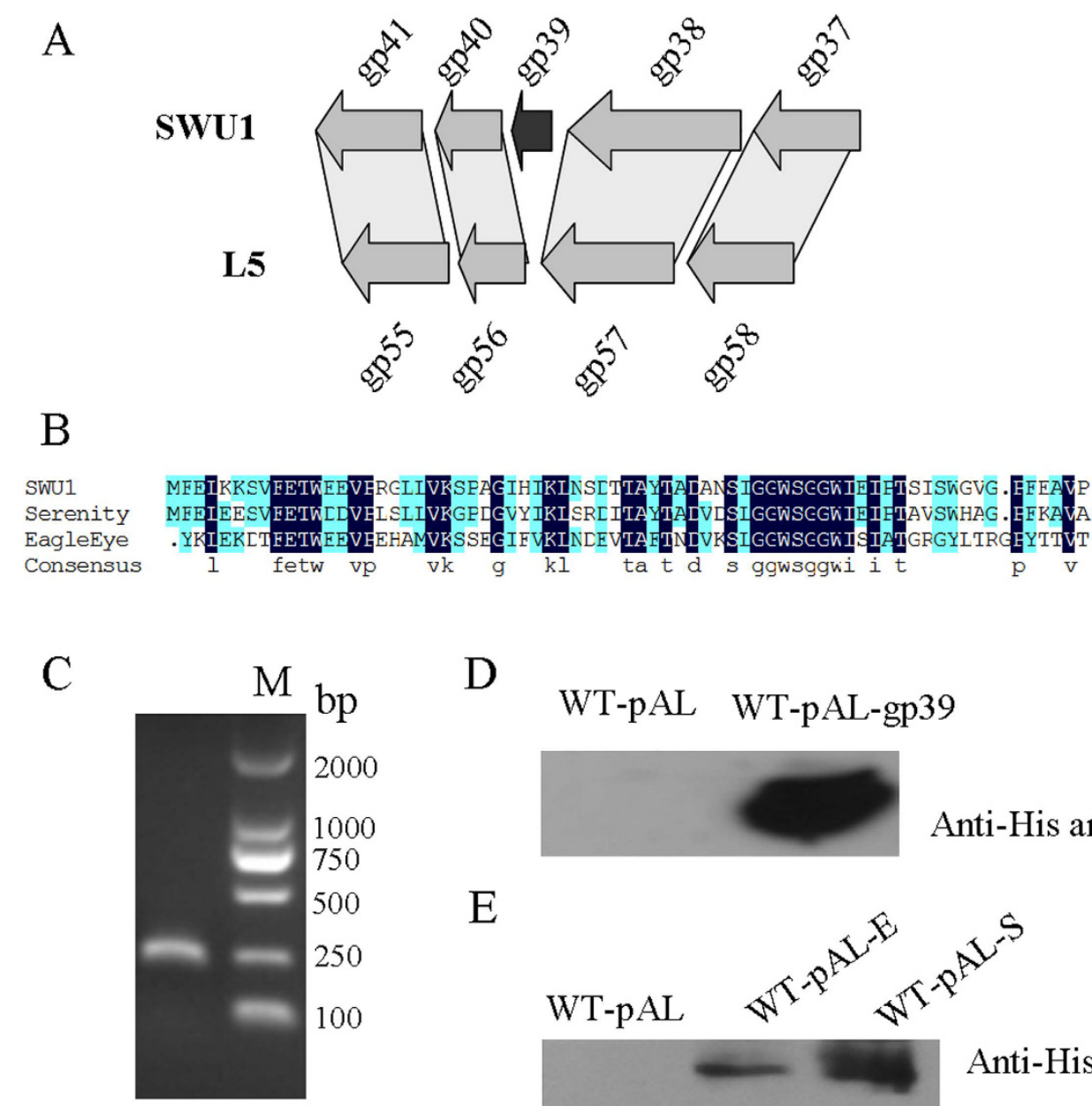

Figure 1. The expression of a novel gene from mycobacteriophage SWU1 in M. smegmatis. (A) Comparison of the organization of the gp39 genome locus in SWU1 and related species L5. The grey shading represents region of conservation between genomes. (B) Amino acid sequence alignment (generated using DNAMAN) of gp39 in SWU1 and close relative. (C) PCR amplification of gp39 encoding sequence approximately $240 \mathrm{bp}$. The SWU1gp39 gene (D) and its homologous genes from Serenity or EagleEye (E) were expressed in M. smegmatis and detected using Western blotting.

Expression of SWU1gp39 in M. smegmatis can potentiate multiple anti-tuberculosis drugs. WT-pAL-gp39 has growth defect in the presence of multiple antibiotics, including isoniazid, erythromycin, norfloxacin and ampicillin. To determine whether expression of SWU1gp39 will compromise the survival during lethal antibiotics stress, we exposed WT-pAL and WT-pAL-gp39 cells to various concentrations of several antimicrobial compounds (Fig. 3). First, we analysed the effect of isoniazid, the survival of WT-pAL-gp39 was 10-100 folds lower than that of WT-pAL (Fig. 3A). Similar phenomenon was observed when WT-pAL and WT-pAL-gp39 were treated with various concentrations of erythromycin for $24 \mathrm{~h}$ and norfloxacin for $4 \mathrm{~h}$ (Fig. 3B,C). These data indicate that SWU1gp39 protein contributes to the lethal activity of isoniazid, erythromycin and norfloxacin against wild type cells. To better understand the role of SWU1gp39 in bacteria upon harsh stress, another three fluoroquinolones were tested on WT-pAL and WT-pAL-gp39. Differential survival rates were found when WT-pAL and WT-pAL-gp39 treated with ciprofloxacin (Fig. 3D) and ofloxacin (Fig. 3E), however no difference was found when treated with moxifloxacin (Fig. 3F). Kanamycin, an aminoglycoside antibiotic, kill bacteria dependent on ROS, but no differences were found when WT-pAL and WT-pAL-gp39 were treated with kanamycin. To discriminate whether SWU1gp39 affects cell wall or membrane stress in mycobacteria, vancomycin, another antibiotic blocking cell wall synthesis, was tested. Vancomycin binds to the terminal D-ala-D-ala of the pentapeptide chain on the peptidoglycan precursor molecules on the outside of the cell membrane, thus affecting only the cell wall synthesis machinery in bacteria. Killing curve experiments revealed WT-pAL-gp39 are more susceptibility towards vancomycin than WT-pAL, indicating that SWU1gp39 most likely affects the cell wall integrity.

To determine the sensitivity of WT-pAL-gp39, MICs of WT-pAL and WT-pAL-gp39 were determined in $7 \mathrm{H} 9$ liquid medium at $37^{\circ} \mathrm{C}$ using the broth dilution method containing $0.25 \%$ acetamide. The results confirmed that WT-pAL-gp39 is sensitive to isoniazid, norfloxacin, ciprofloxacin, ofloxacin, nalidixic acid, erythromycin 
A

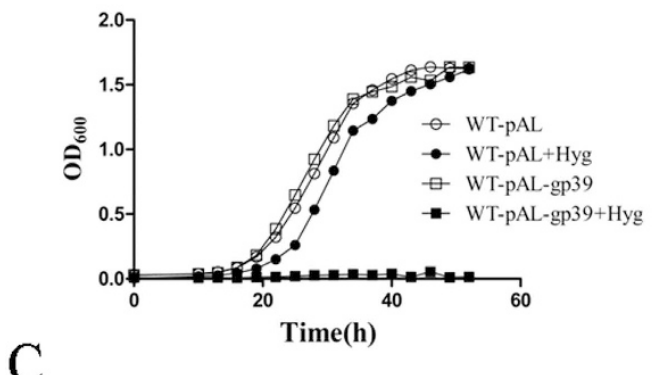

C

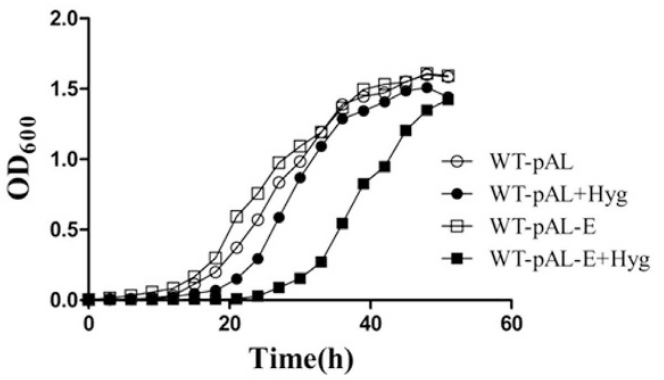

B

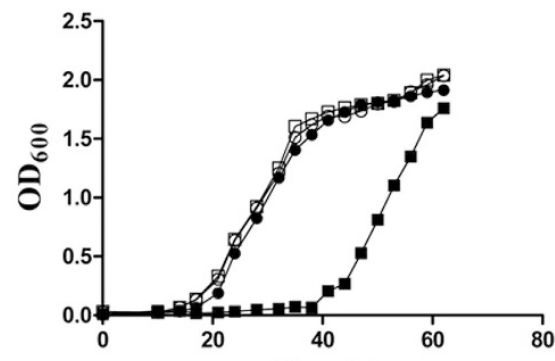

Time(h)

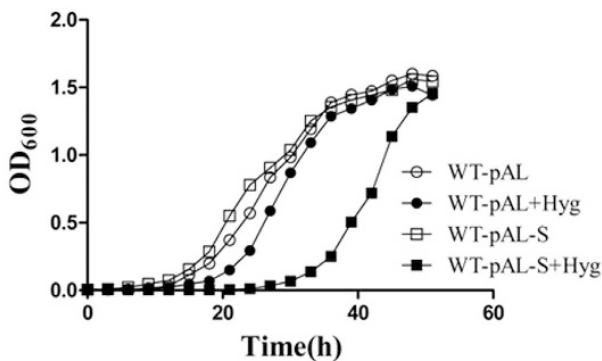

$\mathrm{E}$

Dilution factor $\times 10$

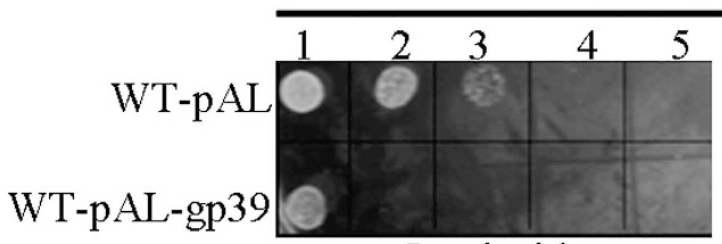

Isoniazide

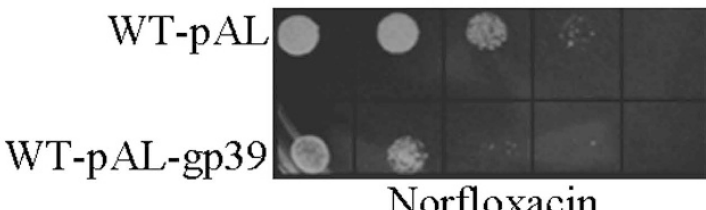

Norfloxacin

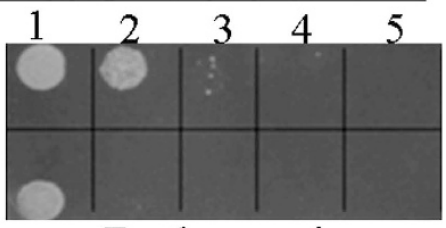

Erythromycin

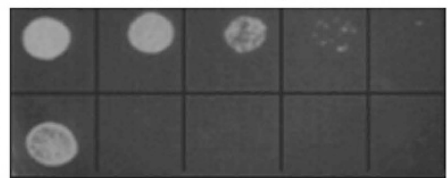

Ampicillin

Figure 2. WT-pAL-gp39 increased bacterial growth defect following exposure to multiple antibiotics. The bacterial strains of WT-pAL, WT-pAL-gp39 (A), WT-pAL-E (C) and WT-pAL-S (D) were grown in M9 medium (B) or Middlebrook 7H9 medium supplemented with $0.05 \%$ Tween $80,0.2 \%$ glycerinum and $0.25 \%$ acetamide, with or without hygromycin $(100 \mu \mathrm{g} / \mathrm{ml})$. The $\mathrm{OD}_{600}$ were determined at an interval of $3 \mathrm{~h}$. (E) Tenfold serial dilutions of WT-pAL and WT-pAL-gp39 were spotted on Middlebrook 7H10 containing isoniazide $(4 \mu \mathrm{g} / \mathrm{ml})$, erythromycin $(16 \mu \mathrm{g} / \mathrm{ml})$, norfloxacin $(1 \mu \mathrm{g} / \mathrm{ml})$ and ampicilin $(100 \mu \mathrm{g} / \mathrm{ml})$. Then the result was recorded when incubated at $37^{\circ} \mathrm{C}$ for 3 days. The data reported represent the means $(n=3) \pm \mathrm{SD}$ (standard deviation).

(Table 1). MICs of WT-pAL-gp39 are between 2- and 8-fold lower than WT-pAL for these antibiotics (Table 1). Sensitivity of WT-pAL-gp39 to other antibiotics, including moxifloxacin, kanamycin, capreomycin, remained unchanged.

Gp39 expression leads to increased $M$. smegmatis sensitivity to various environmental stresses. Environment stress factors are important for mycobacterium growth, the success of $M$. tuberculosis within host necessitates handling of various environment stresses, such as low $\mathrm{pH}$, oxidative stresses. To determine whether gp39 expression also affects the bacterial response to common environmental stresses, we compared the response of WT-pAL-gp39 strain and empty vector control strain to various stresses. The WT-pAL-gp39 strain showed reduced survival following exposure to $52^{\circ} \mathrm{C}$ for $20 \mathrm{~min}$ (Fig. 4A) and low pH for $6 \mathrm{~h}$ (Fig. 4B) in contrast to the empty vector control. To determine the effect of hydrogen peroxide on WT-pAL-gp39 and WT-pAL, disk diffusion was used (Fig. 4C). The WT-pAL-gp39 strain was more sensitive to redox stress via hydrogen peroxide treatment relative to WT-pAL, as the zone of inhibition was significantly larger for the SWU1gp39 expression when compared to vector control (Fig. 4D). Taken together, these results showed that SWU1gp39 expression increased M. smegmatis sensitivity to various environment stresses. 
A

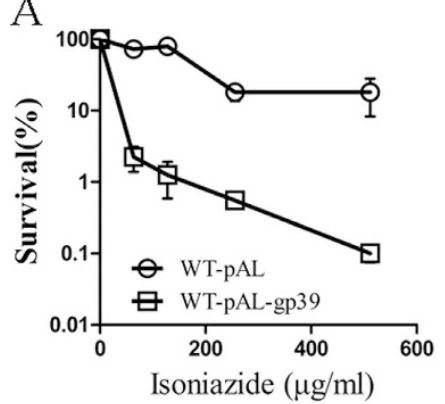

$\mathrm{D}$

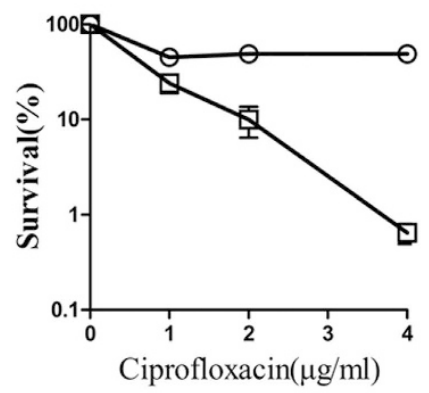

G

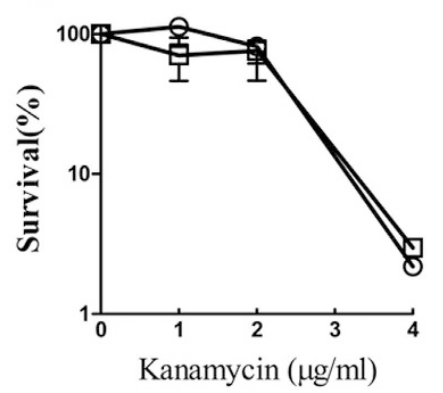

$\mathrm{B}$

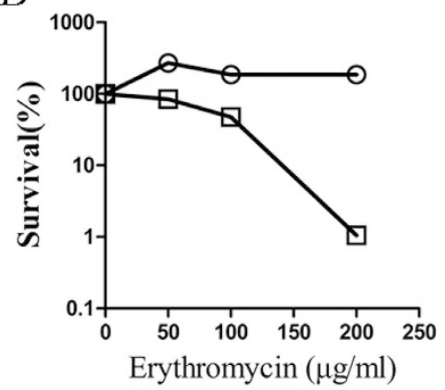

$\mathrm{E}$

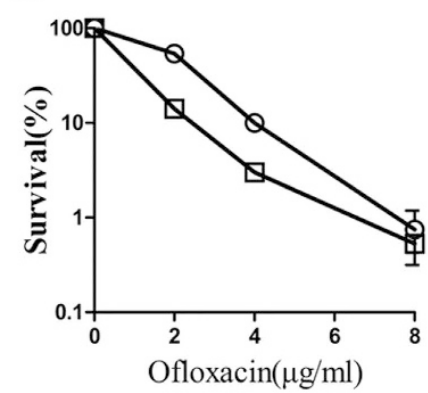

$\mathrm{H}$

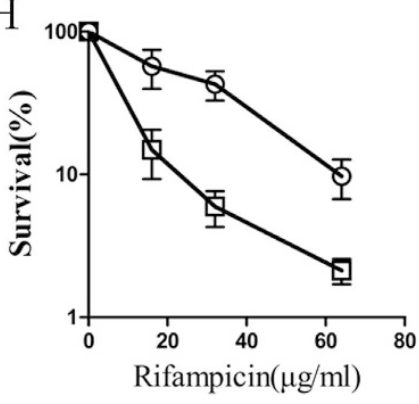

$\mathrm{C}$

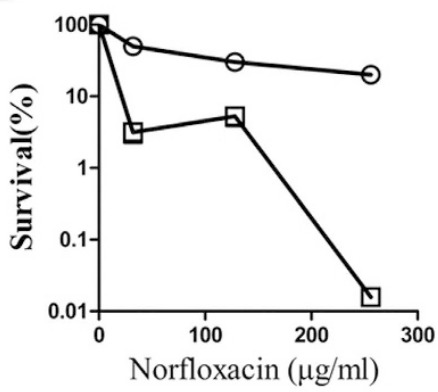

F
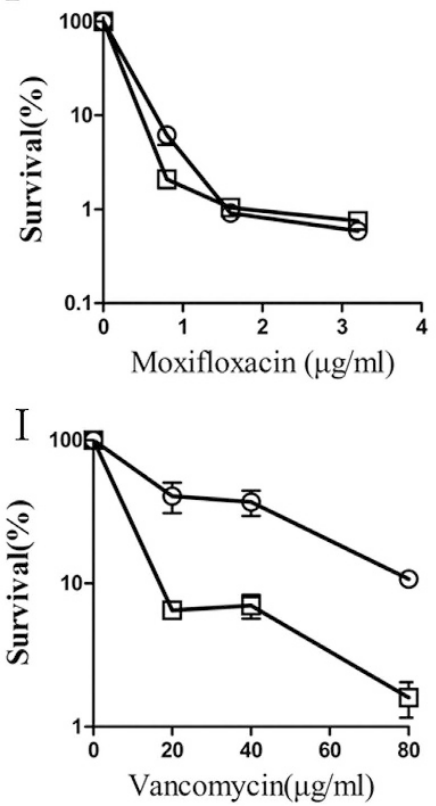

Figure 3. SWU1gp39 expression reduced bacterial survival following exposure to lethal stress. The control strain $\left(\mathrm{WT}-\mathrm{pAL}, \mathrm{OD}_{600}=1\right)$ and recombinant strain $\left(\mathrm{WT}-\mathrm{pAL}\right.$-gp39, $\left.\mathrm{OD}_{600}=1\right)$ were diluted in $7 \mathrm{H} 9$ medium and then treated with isoniazid for $12 \mathrm{~h}(\mathbf{A})$, erythromycin for $24 \mathrm{~h}(\mathbf{B})$, norfloxacin for $4 \mathrm{~h}(\mathrm{C})$, ciprofloxacin for $4 \mathrm{~h}(\mathbf{D})$, ofloxacin for $4 \mathrm{~h}(\mathbf{E})$, moxifloxacin for $2 \mathrm{~h}(\mathbf{F})$, kanamycin for $2 \mathrm{~h}(\mathbf{G})$, rifampicin for $12 \mathrm{~h}(\mathbf{H})$ or vancomycin for $10 \mathrm{~h}(\mathbf{I})$ as the indicated concentrations. Symbols: empty circle, WT-pAL; empty squares, WT-pAL-gp39. Experiments were performed three times and similar results were obtained, error bars indicate standard deviation.

\begin{tabular}{|c|c|c|c|c|c|c|c|c|c|c|c|c|c|c|}
\hline \multirow[b]{2}{*}{ Strain } & \multicolumn{14}{|c|}{$\operatorname{MIC}(\mu \mathrm{g} / \mathrm{ml})$} \\
\hline & INH & NOR & CIP & MOX & OFL & NAL & ERY & KAN & RIF & VAN & AMP & STR & CAP & CHL \\
\hline WT-pAL & 8 & $8-16$ & 1 & 0.2 & 0.5 & 256 & 100 & 0.25 & 4 & 2.5 & 1600 & 0.5 & 1.25 & 32 \\
\hline WT-pAL-gp39 & 4 & 2 & 0.25 & 0.2 & 0.25 & 128 & 25 & 0.25 & 4 & 1.25 & 800 & 0.5 & 1.25 & 32 \\
\hline
\end{tabular}

Table 1. MIC of various antibiotics for WT-pAL and WT-pAL-gp39. INH, isoniazid; NOR, norfloxacin; CIP, Ciprofloxacin; MOX, Moxifloxacin; OFL, Ofloxacin; NAL, Nalidixic acid; ERY, Erythromycin; KAN, Kanamycin; RIF, Rifampicin; VAN, Vancomycin; AMP, Ampicillin; STR, Streptomycin; CAP, Capreomycin; CHL, Chloramphenicol.

Gp39 expression regulates the transcription of multiple $M$. smegmatis genes. In order to gain insight into the regulatory changes in M. smegmatis expressed SWU1 gp39, we compared the global transcription alteration of the recombinant strain (WT-pAL-gp39) and the empty vector (WT-pAL) control strain during mid-log phase by using RNA-Seq transcriptome sequencing. A total of 867 genes (details are listed in the Supplementary materials) showed significant differential transcription in WT-pAL-gp39, including 511 downregulated genes and 356 upregulated genes. Based on gene ontology and KEGG analysis, the recombinant strain showed differential expression of a wide array of genes involved in metabolic process (Fig. 5). SWU1gp39 expressed in M. smegmatis potentiated multiple anti-tuberculosis drugs lethality, but few genes were found to be directly involved in antibiotics except MSMEG_6440 (Rv3854c, ethA), MSMEG_5312, 
A

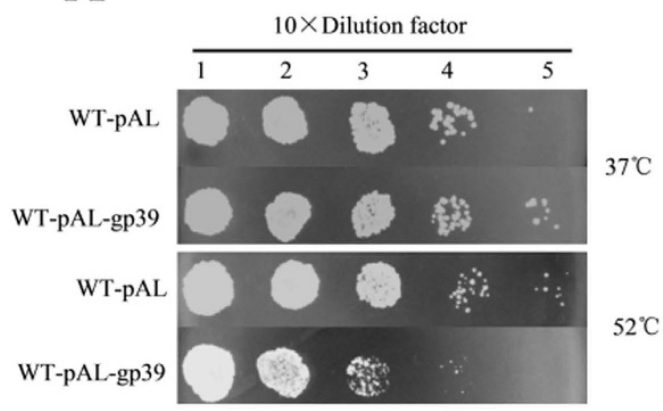

$\mathrm{C}$ WT-pAL-gp39

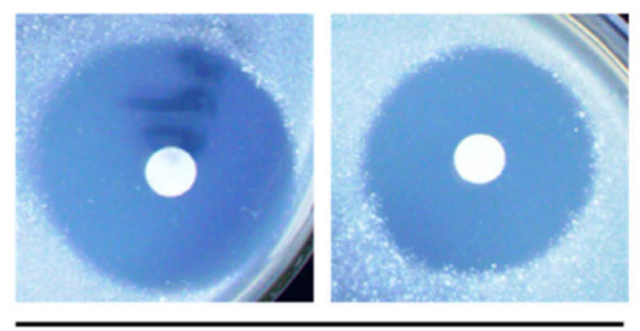

$1 \% \mathrm{H}_{2} \mathrm{O}_{2}$
$\mathrm{B}$

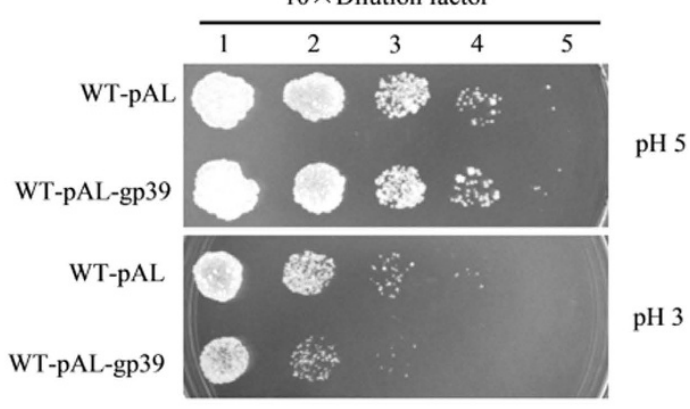

$\mathrm{D}$

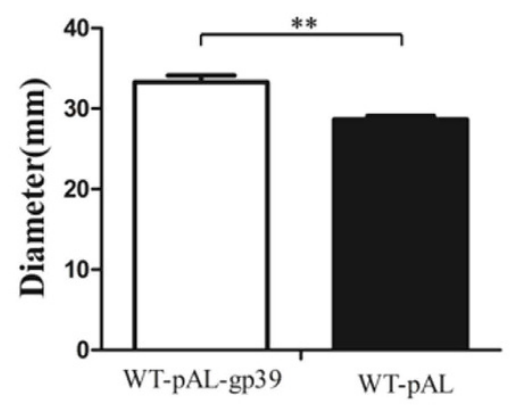

Figure 4. Gp39 expression leads to increased M. smegmatis sensitivity to various environmental stresses. (A) Survival of WT-pAL and WT-pAL-gp39 after treatment with heat shock. (B) Survival of WT-pAL and WT-pAL-gp39 after treatment with low $\mathrm{pH}$. (C) Disc diffusion assay was performed using discs containing $1 \% \mathrm{H}_{2} \mathrm{O}_{2}$. (D) The diameter zone of complete inhibition was measured. The data reported represent the means $(\mathrm{n}=3) \pm \mathrm{SD}$.

MSMEG_1420 and MSMEG_5102 (Table 2). ETH is a prodrug, activated by the NADPH-specific flavin ademine dinucleotide-containing monooxygenase EthA (MSMEG_6440 or Rv3854c). We found that WT-pAL-gp39 showed some growth defect on the solid medium containing indicated concentration of ETH (Fig. S1). This might be due to upregulated EthA expression or more activated ETH. To our knowledge, these genes can not explain why WT-pAL-gp39 became more sensitive to multiple antibiotics. Interestingly, the WT-pAL-gp39 significantly downregulated genes involved in lipid metabolism, including fadD, acpM, kasA, kasB, accD6 (Table 2). Mycolic acids are major and specific long-chain fatty acids essential for the M. tuberculosis cell envelope. The biosynthesis of mycolic acid precursors requires two types of fatty acid synthesis (FASs), the eukaryotic-like multifunctional enzyme FAS I and the acyl carrier protein (ACP)-dependent FAS II systems (Fig. 5D). The genes encoding the FAS II enzymes are distributed in three independent transcription units ${ }^{25}$. The first is formed by fabD-acpM-kasA-kasB-accD6 (Fig. 5D), and all of them are downregulated in WT-pAL-gp39. MabR (mycolic acid biosynthesis regulator) was originally identified as a putative transcriptional regulator encoded by MSMEG_4324 (Rv2242) and located immediately upstream of the main Fas II operon. Overexpression of MabR in $M$. smegmatis represses the transcription of $f a d \mathrm{D}, \operatorname{acpM}, k a s \mathrm{~A}, k a s \mathrm{~B}$ and was accompanied by reduced levels of mycolic acids ${ }^{26}$. Several genes involved in lipid degradation were also upregulated in WT-pAL-gp39 (Table 2). These results suggested that SWU1gp39 might affect the biosynthesis of cell wall mycolic acids components.

Gp39 expression induces redox-related physiological alterations. Our previous transcriptome of SWU1gp39 recombinant strain was associated with altered global metabolism. Antibiotics lethality was reported to be related with redox-related physiological alterations ${ }^{27}$. In order to characterize the redox potential of SWU1gp39 recombinant strain and vector, DCFH-DA was used to monitor the ROS level. DCFH-DA readily penetrates bacterial cells ${ }^{28,29}$; once entry the cells, DCFH-DA is converted by cellular esterases into a nonmembrane-permeating compound that can be oxidized to a fluorescent form by $\mathrm{ROS}^{28}$. Bacterial cell were treated with $10 \mu \mathrm{M}$ DCFH-DA and then fluorescence intensity were detected with excitation at $488 \mathrm{~nm}$ and emission at $515 \mathrm{~nm}$. As shown in Fig. 5B, the recombinant strain exhibited higher fluorescence labeling than the vector control strain. The ROS levels between WT-pAL-gp39 and WT-pAL were also confirmed by second method described below.

As FAS II utilize NADH as cofactors and multiple NAD+ metabolism related genes were downregulated in recombinant strain based on transcriptomic data (see TABLE S1 in the supplemental material), expression of SWU1gp39 in M. smegmatis might change the cellular NAD+/NADH level. Therefore, the cellular NAD + and $\mathrm{NADH}$ level were measured. The results showed that SWU1gp39 expression decreased the cellular NAD + level 
A

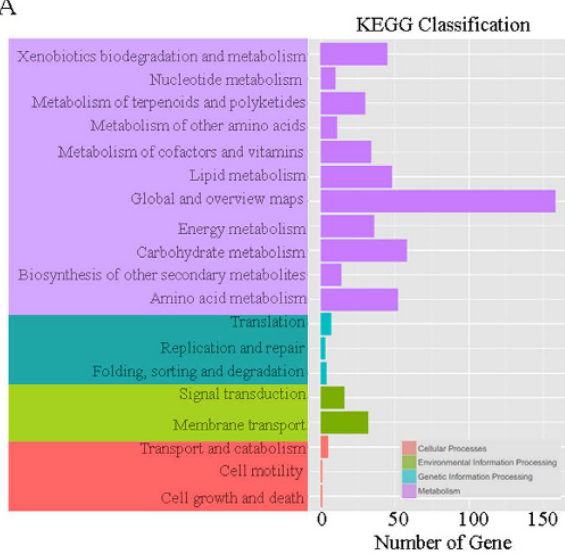

B
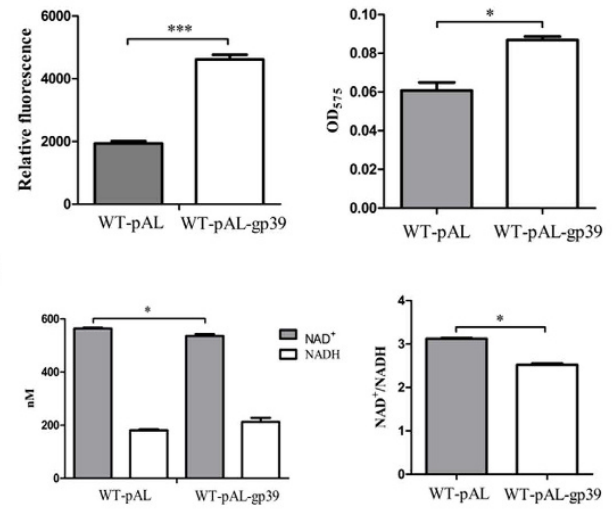

$\mathrm{D}$

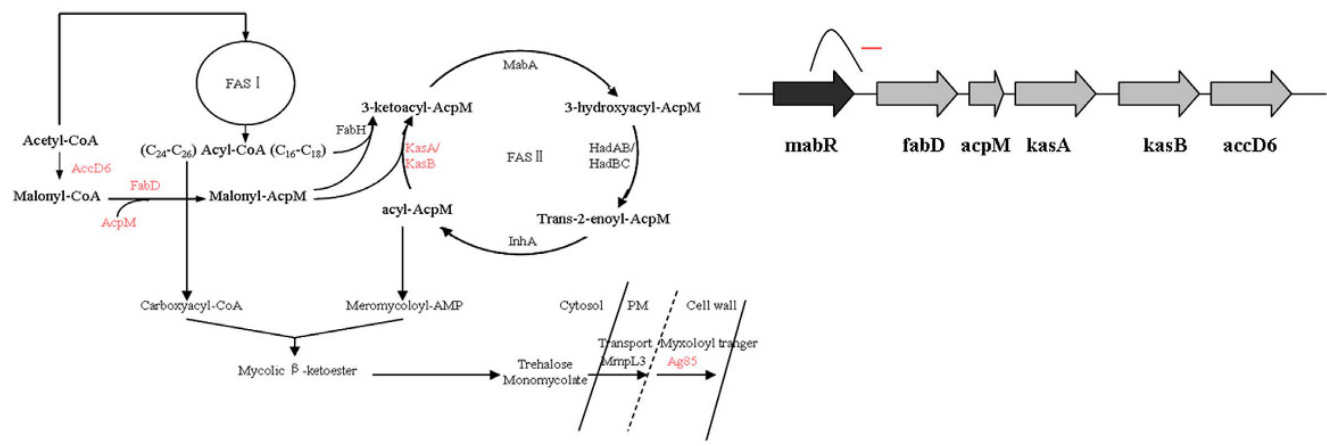

Figure 5. (A) KEGG classification analysis of transcriptional data of WT-pAL and WT-pAL-gp39.

(B) ROS determined in WT-pAL and WT-pAL-gp39. The data reported represent the means $(\mathrm{n}=3) \pm \mathrm{SD}$.

(C) Comparison of NAD ${ }^{+}$and NADH levels in WT-pAL and WT- pAL-gp39. The data reported represent the means $(n=3) \pm S D$. (D) The FAS I and FAS II pathways in mycobacteria.

in WT-pAL-gp39 cells in comparison to WT-pAL (Fig. 5C). Meanwhile, the level of NADH in WT-pAL-gp39 cells was slightly higher than that in WT-pAL. The ratio of NAD+/NADH was $20 \%$ lower in WT-pAL-gp39 than that in WT-pAL (Fig. 5C).

Gp39 expression affects surface motility and biofilm formation. Because the SWU1gp39 expression showed susceptibility to multiple antibiotics targeting cell wall, we determined whether gp39 can affect the sliding motility and biofilm formation. Surprisingly, WT-pAL-gp39 exhibit enhanced sliding on the surface of minimum medium agar plates in comparison with WT-pAL (Fig. 6A,B). In some pathogenic bacteria, such as Vibrio cholerae, Pseudomonas aeruginosa, Haemophilus influenza, Streptococcus sp., Escherichia coli and M. tuberculosis, host persistence and antibiotic tolerance are closely correlated with the ability to form biofilms ${ }^{30}$. At day 3 after inoculation, the culture of control strain has already colonized the whole surface of the liquid medium, whereas the recombinant strain only formed sporadic clumps in the liquid phase and a few islands of growth on the surface (Fig. 6C). These results are reminiscent of an effect of SWU1gp39 on the cell wall of mycobacterium. Scanning electron microscopy did not show strikingly difference between WT-pAL-gp39 and WT-pAL, but showed more pits in the surface of WT-pAL-gp39 (Fig. 7A-D and Fig. S2). Consistent with a defective cell wall, WT-pAL-gp39 became more sensitive to the detergent sodium dodecyl sulphate (SDS) upon treatment with different time and concentration (Fig. 7E,F). WT-pAL-gp39 was at least 10-100 folds more susceptible to SDS than WT-pAL.

The cell wall permeability of $\boldsymbol{M}$. smegmatis Gp39 recombiant is altered. $\quad$ M. smegmatis with SWU1gp39 expression was more sensitive to various hydrophobic antibiotics, including erythromycin, norfloxacin, ciprofloxacin, ofloxacin, and rifampicin, as well as isoniazid and vancomycin which are hydrophilic (Fig. 3). However, the sensitivity to other hydrophobic or hydrophilic antibiotics, moxifloxacin, kanamycin, streptomycin, capreomycin and chloramphenicol, remained unchanged (Table 1).

To examine whether the increased drug sensitivity of the abovementioned recombinant bacteria is caused by a general increase in cell permeability, we used fluorescence spectroscopy to measure the whole-well accumulations of ethidium bromide and Nile Red, representatives of the hydrophilic and hydrophobic compounds, respectively ${ }^{31}$. The results showed that both compounds accumulated more rapidly and to higher levels in the recombinant strain than the empty vector control strain, indicating an increase in cell wall permeability (Fig. 8A,B). 


\begin{tabular}{|c|c|c|c|c|}
\hline Category & Gene ID & Fold change & Homologs & Gene description \\
\hline \multirow{4}{*}{ Drug resistance related genes } & MSMEG_6440 & 16.9 & Rv3854c & monooxygenase, flavin-binding family protein \\
\hline & MSMEG_5312 & 2.59 & & multidrug ABC transporter ATPase \\
\hline & MSMEG_1420 & 2.42 & & transcriptional regulatory protein \\
\hline & MSMEG_5102 & 3.22 & & $\mathrm{ABC}$ transporter ATP-binding protein \\
\hline \multirow{20}{*}{ lipid metabolism } & MSMEG_4324 & 7.45 & Rv2242 & MabR, mycolic acid biosynthesis regulator \\
\hline & MSMEG_4327 & -4.82 & Rv2245 & KasA, involved in meromycolate extension \\
\hline & MSMEG_4326 & -4.86 & Rv2244 & AcpM, lipid metabolism \\
\hline & MSMEG_4328 & -4.14 & Rv2246 & KasB, lipid metabolism \\
\hline & MSMEG_4325 & -3.75 & Rv2243 & FadD, lipid metabolism \\
\hline & MSMEG_4329 & -3.50 & Rv2247 & AccD6, lipid metabolism \\
\hline & MSMEG_3580 & -2.70 & Rv0129c & lipid metabolism \\
\hline & MSMEG_6179 & -2.12 & Rv3667 & lipid metabolism \\
\hline & MSMEG_2078 & -2.01 & Rv1886c & lipid metabolism \\
\hline & MSMEG_1387 & 3.64 & Rv0672 & involved in lipid degradation \\
\hline & MSMEG_1388 & 4.16 & Rv0673 & enoyl-CoA hydratase EchA4 \\
\hline & MSMEG_1390 & 3.39 & Rv0675 & enoyl-CoA hydratase EchA5 \\
\hline & MSMEG_5996 & 3.24 & Rv3546 & involved in lipid degradation \\
\hline & MSMEG_4717 & 3.00 & Rv2502c & fatty acid metabolism \\
\hline & MSMEG_6041 & 2.41 & Rv3573c & lipid degradation \\
\hline & MSMEG_3465 & 2.18 & Rv1925 & lipid degradation \\
\hline & MSMEG_4716 & 2.51 & Rv2501c & lipid metabolism \\
\hline & MSMEG_5907 & 2.15 & Rv3505 & involved in lipid degradation \\
\hline & MSMEG_5923 & 2.10 & Rv3523 & lipid carrier protein \\
\hline & MSMEG_5922 & 2.18 & Rv3522 & lipid transfer protein \\
\hline
\end{tabular}

Table 2. Genes differentially regulated in WT-pAL- $g$ p39 compared with WT-pAL.

Mycolic acids are major and specific long-chain fatty acids essential for mycobacterium dell envelope. Mycobacteria possess two fatty acid synthase systems, the eukaryotic-like fatty acid synthase typeI (FAS I) and the prokaryotic FAS II. Mycobacterial FAS I synthesizes fatty acids in a bimodal pattern, which are elongated by the FAS II system to produce mycolic acids, long-chain fatty acids, which are the major constituents of the mycobacterial cell wall. FAS I and FAS II utilize NADPH and NADH as cofactors. Our data showed that multiple genes encoding the FAS II enzymes were downregulated and NADH cofactor accumulated, suggesting that FAS II might be affected by SWU1gp39. To explore whether the expression of SWU1gp39 in M. smegmatis can change the composition of fatty acids, gas chromatography mass spectrometry (GC-MS) was used to detect the fatty acids of WT-pAL-gp39 and WT-pAL. There are 14 major compounds identified in WT-pAL-gp39 and WT-pAL, from C7 to C24 (Fig. 8C). Several compounds were found accumulated in WT-pAL-gp39, especially C7:0, C8:0, C11:0, C14:0, MeC15:0, C18:1 $\omega 5$ (c), C24:0 (Fig. 8C). The data indicated that SWU1gp39 can alter the relative abundance of fatty acids in M. smegmatis.

\section{Discussion}

Multidrug-resistant M. tuberculosis (MDR-MTB) and extensively drug-resistant M. tuberculosis (XDR-MTB) represent formidable challenge to global public health. New effective drug or small-molecule potentiators of bacteriocidal antibiotics are urgently needed ${ }^{32}$. Peptidoglycan recognition proteins (PGRPs) are a family of evolutionary conserved antibacterial innate immunity proteins ${ }^{33}$. PGRPs kill antibiotic-resistant bacteria, synergistic targeting of oxidative, thiol, and metal stress can be used for the development of new approaches to kill antibiotic resistant bacteria ${ }^{34}$. Vitamin $\mathrm{C}(\mathrm{VC})$ is an essential nutrient for some mammals and potent antioxidant. The activity of VC against drug-susceptible, MDR- and extensively drug-resistant (XDR) M. tuberculosis was associated with the presence of high iron concentration, reactive oxygen species (ROS) production and DNA damage. Phage, as a nemesis of the bacteria, is a useful tool for scientific research. Phages were highly expected for the treatment of drug-resistant bacteria.

In this study, we showed that SWU1gp39 expressed M. smegmatis showed change of sensitivity to multiple antibiotics, including isoniazid (INH), erythromycin (ERY), fluoroquinolones, rifampicin (RIF), vancomycin (VAN), ampicillin (AMP) when. Isoniazid is a pro-drug, targeting enoyl ACP reductase InhA after activated by the catalase-peroxidase $\mathrm{KatG}^{10}$. Erythromycin is a macrolide antibiotic targeting the $50 \mathrm{~S}$ ribosome and inhibiting bacterial protein synthesis ${ }^{35}$. Fluoroquinolones is a class of synthetic drugs, which target the gyrase (gyrA or gyrB) and also function via the secondary effect of lethal doses of hydroxyl radicals produced ${ }^{36}$. Rifampicin is first-line anti-tuberculosis drug, inhibiting the transcription of $M$. tuberculosis by binding to the beta subunit of the RNA polymerase (RpoB) encoded by the $r p o B$ gene $^{37,38}$. Rifampicin also induced hydroxyl radical formation $^{39}$. Vancomycin and ampicillin inhibit bacteria growth by targeting bacteria cell wall and also lead to the production of hydroxyl radical ${ }^{40}$. Our transcriptome data showed that the transcription of most genes associated with antibiotics action did not changed by gp39 (Table 2), suggesting a negligible role of WT-pAL-gp39 in regulating the transcription of genes known to be associated with antibiotics action. WT-pAL-gp39 showed growth defect in 
A

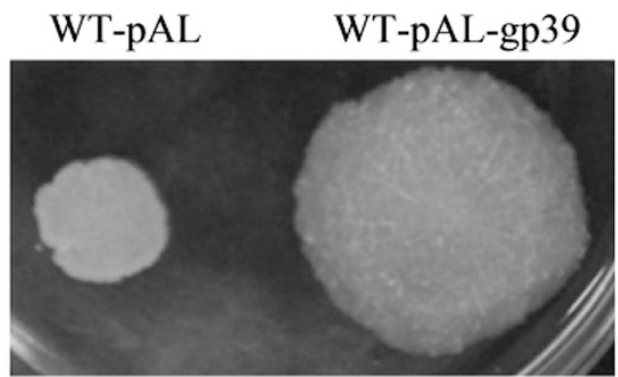

B

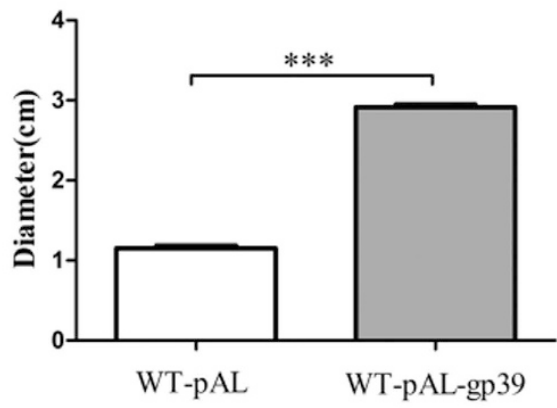

C

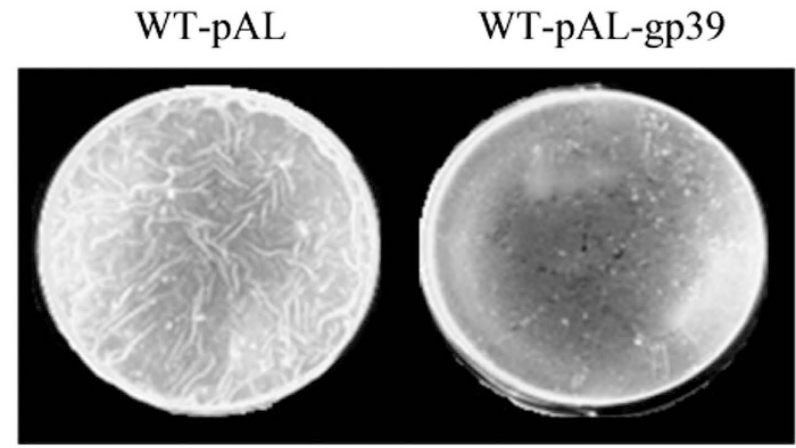

Figure 6. Effect of SWU1gp39 on surface motility and biofilm growth. (A) Sliding motility of M. smegmatis on M63 medium. (B) The diameter zome of the motility colony. The data reported represent the means $(\mathrm{n}=3) \pm \mathrm{SD}$. (C) Surface biofilm growth of M. smegmatis strain.

the presence of hygromycin in comparison to WT-pAL even though pALACE contains a hygromycin resistance cassette. We speculated that WT-pAL-gp39 possess higher cell wall permeability in comparison with WT-pAL.

The intracellular survival of $M$. tuberculosis entails extraordinary capability to tackle diverse stresses. Our data showed that WT-pAL-gp39 can weaken the Mycobacteria ability to handle multiple environment stresses including low $\mathrm{pH}$, heat shock and hydrogen peroxide, which are relevant to antibiotics efficacy ${ }^{41,42}$. Cell wall defect can be found in some mutants, such as acid-sensitive mutants ${ }^{43}$, exopolyphosphatase Rv1026/PPX2 ${ }^{44}$ and lipoprotein mutation ${ }^{45}$. The survival ability of WT-pAL-gp39 was 10- to 100-fold reduced in comparison to that of WT-pAL cells when exposed to SDS for various times or various concentration, suggesting cell wall defect in WT-pAL-gp39. Multiple antibiotics targeting cell wall were discovered, it is tempting to explore whether these cell wall targeting antibiotics have similar sensitizing effect. Congo red, a dye binding to the lipoproteins present on the mycobacterial surface ${ }^{46}$, was used to characterize modifications affecting the cell wall ${ }^{45}$. After 4 days of growth on a solid medium containing Congo Red at $37^{\circ} \mathrm{C}$ (without Tween 80 ), no difference was observed between the two M. smegmatis strains (Fig. S3). Biofilm plays crucial role in antibiotic tolerance and persistence in M. tuberculosis ${ }^{47}$. In this study, we found that SWU1gp39 expression was associated with reduced biofilm formation, but increased in surface motility. This is consistent with previous results that WT-pAL-gp39 antibiotic susceptibility is associated with the environment stressor and biofilm, but not Congo Red uptake.

This work showed for the first time that mycobacteriophage SWU1gp39 protein altered the cell wall permeability, manifested by increased uptake of the polar compound $\mathrm{EtBr}$ and the lipophilic dye Nile red. The intrinsic resistance of mycobacteria to most antimicrobial agents is generally attributed to an unusual cell wall dominated by lipids and carbohydrates, which provides a barrier to noxious compounds and limits drug uptake ${ }^{48,49}$. Previous studies have shown that mycobacterial uptake of Nile red is directly related to cell wall lipid components and susceptibility to lipophilic drugs ${ }^{50,51}$. Mycobacterium cell wall permeability for EtBr also is closely related with antibiotic resistance ${ }^{52}$. We conclude that WT-pAL-gp39 can sensitize the recombinant to multiple stresses via increasing the cell wall permeability. More accumulation of short-chain fatty acids in WT-pAL-gp39 than in the WT-pAL suggested that SWU1gp39 disrupted normal synthesis of long-chain fatty acids, the major components of mycobacteria cell wall.

Phage therapy was renewed as promising alternative to antibiotics for tackling resistant pathogens ${ }^{53}$. Engineered bacteriophage with protein targeting gene networks overexpressed can boost the bactericidal effect of antibiotics ${ }^{54}$. For therapeutic applications of phage, SWU1gp39 might be used as a broad-spectrum antibiotic adjuvant or potentiator, in addition to be included in engineered bacteriophage to enhance bacterial killing by antibiotics, especially first-line anti-tuberculosis drugs such as isoniazed and rifampicin. 

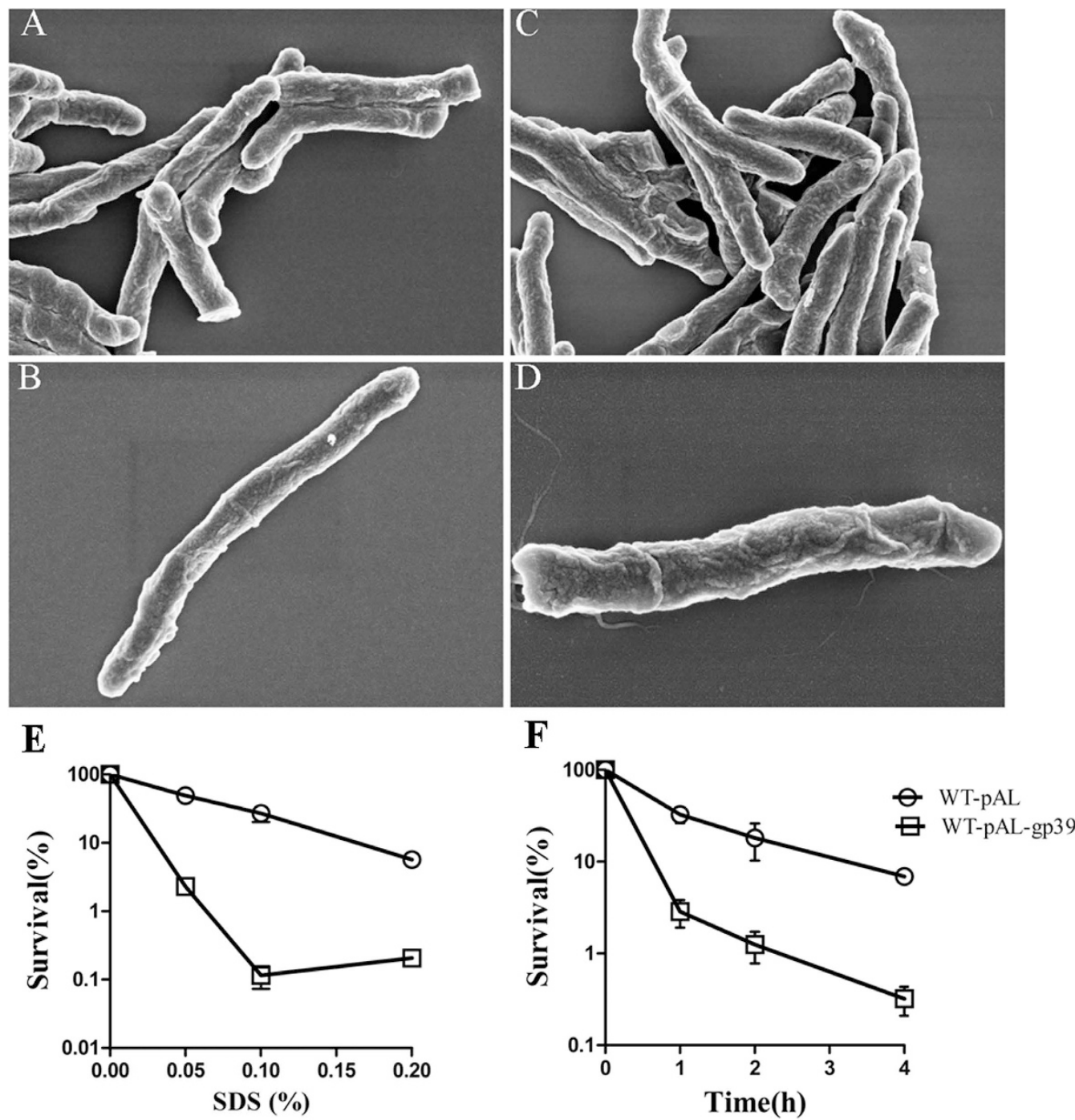

Figure 7. Scanning electron micrographs of WT-pAL (A,B) and WT-pAL-gp39 (C,D). The effects of SWU1gp39 on bacterial survival after treatment with SDS as indicated concentration (E) and time (F). Symbols: empty circle, WT-pAL; empty squares, WT-pAL-gp39. Experiments were performed three times and similar results were obtained, error bars indicate standard deviation.

A

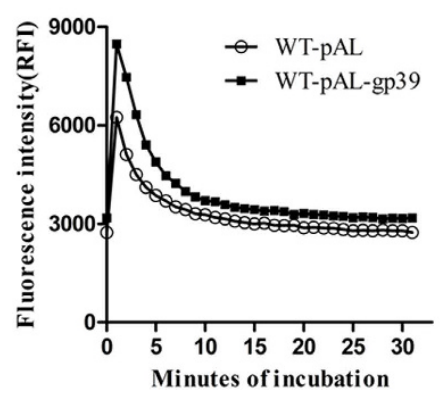

B

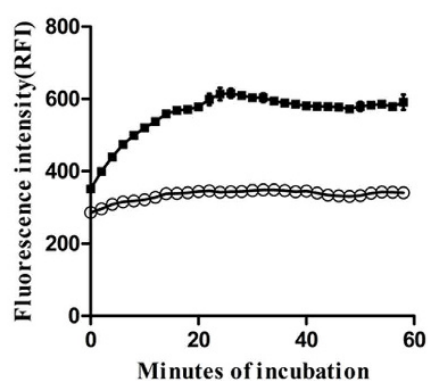

$\mathbf{C}$

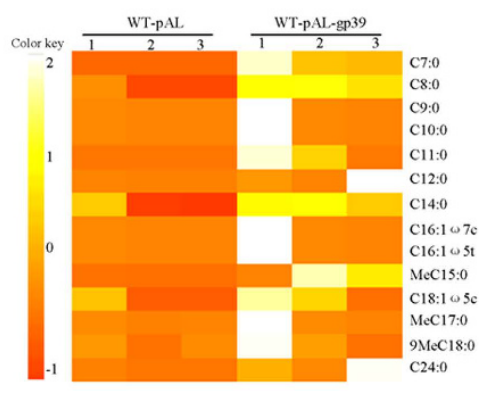

Figure 8. SWU1gp39 expression in M. smegmatis results in increased cell wall permeability. (A) Mid-logphase cultures of WT-pAL $\left(\mathrm{OD}_{600}=0.5\right)$ control and WT- pAL-gp39 $\left(\mathrm{OD}_{600}=0.5\right)$ strains were incubated in PBS with $25 \mathrm{mM}$ glucose and $2 \mu \mathrm{g} / \mathrm{ml}$ ethidium bromide. The data reported represent the means $(\mathrm{n}=3) \pm \mathrm{SD}$. (B) Mid-log-phase cultures of WT-pAL control and WT-pAL-gp39 strains were incubated in PBS containing $25 \mathrm{mM}$ glucose and $2 \mu \mathrm{M}$ Nile red stain. The data reported represent the means $(n=3) \pm S D$. (C) Fatty acid extracted from WT-pAL and WT-pAL-gp39 analyzed by gas chromatography mass spectrometry (GC-MS). Symbols: empty circle, WT-pAL; filled squares, WT-pAL-gp39. 


\begin{abstract}
Methods
Bacteria strain, plasmid, and growth conditions. M. smegmatis $\mathrm{mc}^{2} 155$ and recombinant bacteria grown in Middlebrook 7H9 medium supplemented with $0.05 \%$ Tween 80 and $0.2 \%$ glycerinum or Middlebrook $7 \mathrm{H} 10$ plates supplemented with $0.5 \%$ glycerinum. Luria-Bertani medium was used to culture E. coli strains. Antibiotics were added at following concentrations: ampicillin, $100 \mu \mathrm{g} / \mathrm{ml}$; kanamycin, $50 \mu \mathrm{g} / \mathrm{ml}$ for E. coli and $20 \mu \mathrm{g} / \mathrm{ml}$ for $M$. smegmatis; hygromycin, $75 \mu \mathrm{g} / \mathrm{ml}$ for E. coli or $50 \mu \mathrm{g} / \mathrm{ml}$ for M. smegmatis. All cultures were incubated at $37^{\circ} \mathrm{C}$.
\end{abstract}

Expression of His-tagged SWU1gp39 protein. The open reading frame encoding SWU1 gp39 gene was amplified by PCR from SWU1 genomic DNA using the forward primer $5^{\prime}$ ATGGATCCATGTTCGAACTG3' containing a BamHI site (underlined) and the reverse 5' AATCGATTCACAGGGGCACCGCTT3' containing a ClaI site (underlined). The PCR product of approximately $250 \mathrm{bp}$ was cloned into Mycobacteria-Escherichia coli shuttle plasmid $p A L A C E$. The sequence verity was confirmed by DNA sequencing.

Bacterial growth curves. Recombinant strains WT-pAL and WT-pAL-gp39 were cultured in 7H9 medium containing $0.5 \%$ glycerol, $0.05 \%$ Tween 80 and $0.25 \%$ acetamide until $\mathrm{OD}_{600}$ reached $0.8-1.0$. Then the cultures were reinoculated in fresh $7 \mathrm{H} 9$ medium at the ratio of $1: 1000$ dilution. Cultures were incubated at $37^{\circ} \mathrm{C}$ with shaking through the entire growth phase. Samples were collected at the same growth stage, and the $\mathrm{OD}_{600}$ values were measured every $3 \mathrm{~h}$ after growth initiation. Experiments were performed in triplicates, and the average values were used to generate growth curves.

Survival curves. Mid-exponential phase cultures of WT-pAL and WT-pAL- $g p 39$ were diluted in $7 \mathrm{H} 9$ medium and grown at $37^{\circ} \mathrm{C}$ treated for various concentrations as indicated with antibiotics. After indicated time, surviving cells were estimated by colony formation on drug-free agar. The percentage cfu recovered was determined relative to an untreated control sampled at the time when antibiotics added. All the experiment was repeated at least three times.

Disk diffusion method. The disk diffusion method was used to qualitatively measure the differences in $\mathrm{H}_{2} \mathrm{O}_{2}$ or SDS sensitivities between WT-pAL and WT-pAL-gp39 mycobacterium. Mid-exponential-phase cultures were used to prepare the lawns of cells as previously described ${ }^{55}$. An indicated concentration of $\mathrm{H}_{2} \mathrm{O}_{2}$ or SDS was spotted on $5.5 \mathrm{~mm}$-diameter Whatman filter disks placed on the bacterial lawn. After overnight incubation at $37^{\circ} \mathrm{C}$, the diameter of zone of complete inhibition was measured. All the experiment was repeated at least three times.

Spot tests. WT-pAL and WT-pAL-gp39 were grown to an $\mathrm{OD}_{600}$ of $0.8-1.0$ tested for their susceptibility to antibiotics by spotting a 10 -fold serial dilution initially on Middlebrook $7 \mathrm{H} 10$ containing $0.25 \%$ acetamide and a range of drugs. The concentration of antibiotics for spot tests as following described: isoniazid ( $4 \mu \mathrm{g} / \mathrm{ml})$, erythromycin $(16 \mu \mathrm{g} / \mathrm{ml})$, norfloxacin $(1 \mu \mathrm{g} / \mathrm{ml})$, ampicillin $(100 \mu \mathrm{g} / \mathrm{ml})$.

MIC determination. The MIC of anti-TB drugs were determined as previously described ${ }^{2}$. Briefly, broth dilution with visual inspection of a series of tubes each containing about $10^{5}$ bacteria in $1 \mathrm{ml}$ of $7 \mathrm{H} 9$ medium supplemented with concentrations of drug increasing by 2 times increments and $0.25 \%$ acetamide. Following 3 days incubation at $37^{\circ} \mathrm{C}$, the lowest concentration that prevented visible growth was defined as the MIC.

Heat shock and acid challenge. Mid-exponential phase cultures of WT-pAL and WT-pAL- $g$ p 39 washed with phosphate buffer $(\mathrm{pH} 7.0)$, and then diluted $\mathrm{OD}_{600}=0.5$. For heat shock, the bacteria were incubated in $52^{\circ} \mathrm{C}$ for $20 \mathrm{~min}$, then serially diluted in phosphate buffer and spotted onto $7 \mathrm{H} 9$ plates. For acid challenge studies, the bacteria were incubated in low $\mathrm{pH}$ medium for $6 \mathrm{~h}$, then serially diluted in phosphate buffer and spotted onto 7H9 plates.

RNA-seq. Following exposure of logarithmically growing cultures (WT-pAL and WT-pAL-gp39, $\mathrm{OD}_{600}=0.8$ ) to the inducer acetamide for at least $4 \mathrm{~h}$, RNA was harvested using Trizol-based methods. RNA samples were treated with DNase and purified RNA was got by using RNA clean kit (TIANGEN). The samples were sent to Shanghai Biotechnology Corporation for library construction and sequencing using Illumina Hi seq 2500 (Illumina). The sequence quality of the data sets was checked by Agilent 2100 Bioanalyzer. The transcriptome sequencing (RNA-seq) data were aligned with the $M$. smegmatis $\mathrm{mc}^{2} 155$ genome obtained from NCBI (http://www.ncbi.nlm.nih.gov/genome/?term = MC2_155). Gene expression level was estimated by the reads number. In order to make it comparable between different genes or samples, reads number were converted into FPKM (Fragments Per Kilobase of exon model per Million mapped reads) for the normalization of gene expres$\operatorname{sion}^{56}$. Every gene Fragments number was calculated after TopHat alignment by HTseq ${ }^{57}$, Then normalization was carried out using TMM (trimmed mean of M values) ${ }^{58}$ and the FPKM was calculated by Perl script.

Gene ontology and KEGG classification analysis. Gene Ontology (GO) annotation transcriptome was performed using the UniProt-GOA Database (http://www.ebi.ac.uk/GOA/). Proteins were categorized into biological process, cellular compartment and molecular function according to Gene Ontology annotation. Kyoto Encyclopedia of Genes and Genomes (KEGG) were utilized to annotate pathways: firstly, using KEGG online service tools KAAS to annotate proteins, secondly, using KEGG online service tools KEGG mapper to map on the KEGG pathway database, finally, using InterPro database and InterProScan to annotate protein domains and applying CORUM database to annotate protein complex. 
Scanning electron microscopy study. WT-pAL and WT-pAL-gp39 cells were grown for about $24 \mathrm{~h}$ until the $\mathrm{OD}_{600}$ reaches 1.0 in $7 \mathrm{H} 9$. Cultures were harvested by centrifugation and the harvested pellets were then resuspended in $2.5 \%$ glutaraldehyde solution. The samples were dehydrated in an ascending series of ethanol. After critical point drying, samples were sputtered with platinum and observed by a scanning electron microscopy (SEM; FEI Quanta 200).

Ethidium bromide accumulation/efflux assay and Nile red uptake assays. Strains of mycobacterium were grown in $7 \mathrm{H} 9$ to an $\mathrm{OD}_{600}=1.0$, washed twice with phosphate-buffered saline $(\mathrm{PBS}, 137 \mathrm{mM} \mathrm{NaCl}$, $\left.4.3 \mathrm{mM} \mathrm{Na}_{2} \mathrm{HPO}_{4}, 1.4 \mathrm{mM} \mathrm{KH} \mathrm{PO}_{4}, \mathrm{pH} 7.0\right)$ and resuspended. The resuspended cells were determined and adjusted to 0.4 and containing $25 \mathrm{mM}$ glucose. After standing 5 minutes, $200 \mu \mathrm{l}$ of the suspension cells was added in triplicate to a 96-well black fluoroplate and Nile Red and EtBr were added to final concentrations. The accumulation of these dyes was measured with an excitation of $540 \mathrm{~nm}$ and emission of $630 \mathrm{~nm}$ for Nile Red and an excitation of $545 \mathrm{~nm}$ and emission of $600 \mathrm{~nm}$ for EtBr.

ROS determination in planktonic cells. The intracellular production of ROS was detected by the reduction of nitro blue tetrazolium (NBT) (Sigma) to nitro blue diformazan. Bacterial suspensions ( $500 \mu$ l of stationary phase) were inoculated with $500 \mu \mathrm{l}$ of NBT $(1 \mathrm{mg} / \mathrm{ml})$ at $37^{\circ} \mathrm{C}$ for $30 \mathrm{~min}$. Bacterial cells were separated from the supernatant by centrifugation at $1500 \mathrm{~g}$ for $10 \mathrm{~min}$, and then treated with $600 \mu \mathrm{l}$ DMSO and $800 \mu \mathrm{l}$ of PBS, pH 7.0. Reduced NBT was measured as formazan blue at $575 \mathrm{~nm}$.

The second method we used for ROS determination was Reactive Oxygen Species Assay Kit (Beyotime Institute of Biotechnology, China) based on $2^{\prime}, 7^{\prime}$-dichlorodihydrofluorescein deacetate (DCFH-DA) to determine the generation of ROS. We acquired cell samples in logarithmic phase and stained them with $10 \mu \mathrm{M}$ DCFH-DA. We estimated the fluorescence intensity with excitation at $488 \mathrm{~nm}$ and emission at $515 \mathrm{~nm}$.

$\mathrm{NAD}^{+}$and $\mathrm{NADH}$ concentration assay. $\mathrm{NAD}^{+}$and $\mathrm{NADH}$ concentrations were measured using a $\mathrm{NAD}^{+} / \mathrm{NADH}$ Assay kit (BioAssay Systems). M. smegmatis culture was diluted to $\mathrm{OD}_{600}$ of 0.5 and then washed twice with cold phosphate buffer saline (PBS). Cell pellets were resuspended with $100 \mu \mathrm{l}$ of $\mathrm{NAD}^{+}$extraction buffer for $\mathrm{NAD}^{+}$measurement and $100 \mu \mathrm{l}$ of NADH extraction buffer for NADH measurement. Then extracts were heated at $60^{\circ} \mathrm{C}$ for $5 \mathrm{~min}$. Supernatant was collected for the EnzyChrom NAD ${ }^{+} / \mathrm{NADH}$ Assay kit.

Fatty acid analysis. The Mid-exponential phase cultures M. smegmatis fatty acid were extracted as previously described ${ }^{59}$. The concentration of fatty acid was determined by an Agilent 7890A gas chromatograph with 5975C mass selective detector (GC-MSD) equipped with an Agilent 7693A automatic liquid sampler and a DB-5MS capillary column. The detailed analysis procedure was described previously ${ }^{60}$.

Statistical analysis. Data from at least three biological replicates were used to calculate means and standard deviation (SD) for graphing purposes. Statistical analysis employed the unpaired student's $t$ test, asterisks indicate statistically significant difference $(* P<0.05$; $* * P<0.01 ; * * * P<0.001)$.

\section{References}

1. Ren, H. \& Liu, J. AsnB is involved in natural resistance of Mycobacterium smegmatis to multiple drugs. Antimicrobial agents and chemotherapy 50, 250-255 (2006).

2. Li, Q. et al. Proteasome Accessory Factor C (pafC) Is a novel gene Involved in Mycobacterium Intrinsic Resistance to broadspectrum antibiotics-Fluoroquinolones. Scientific reports 5, 11910 (2015).

3. Wolff, K. A. et al. Protein kinase G is required for intrinsic antibiotic resistance in mycobacteria. Antimicrobial agents and chemotherapy 53, 3515-3519 (2009).

4. Bowman, J. \& Ghosh, P. A complex regulatory network controlling intrinsic multidrug resistance in Mycobacterium smegmatis. Molecular microbiology $91,121-134$ (2014).

5. Nandakumar, M., Nathan, C. \& Rhee, K. Y. Isocitrate lyase mediates broad antibiotic tolerance in Mycobacterium tuberculosis. Nature communications 5, 4306 (2014).

6. Lu, P. et al. The cytochrome bd-type quinol oxidase is important for survival of Mycobacterium smegmatis under peroxide and antibiotic-induced stress. Scientific reports 5, 10333 (2015).

7. Berney, M., Hartman, T. E. \& Jacobs, W. R. A Mycobacterium tuberculosis cytochrome bd oxidase mutant is hypersensitive to bedaquiline. mBio. 5, e01275-01214 (2014).

8. Takayama, K., Wang, L. \& David, H. L. Effect of isoniazid on the in vivo mycolic acid synthesis, cell growth, and viability of Mycobacterium tuberculosis. Antimicrobial agents and chemotherapy 2, 29-35 (1972).

9. Zhang, Y., Heym, B., Allen, B., Young, D. \& Cole, S. The catalase-peroxidase gene and isoniazid resistance of Mycobacterium tuberculosis. Nature 358, 591-593 (1992).

10. Banerjee, A. et al. inhA, a gene encoding a target for isoniazid and ethionamide in Mycobacterium tuberculosis. Science 263, 227-230 (1994).

11. Rozwarski, D. A., Grant, G. A., Barton, D. H., Jacobs, W. R. \& Sacchettini, J. C. Modification of the NADH of the isoniazid target (InhA) from Mycobacterium tuberculosis. Science 279, 98-102 (1998).

12. Zhang, Y. \& Yew, W. Mechanisms of drug resistance in Mycobacterium tuberculosis [State of the art series. Drug-resistant tuberculosis. Edited by Chiang, C. Y. Number 1 in the series]. The International Journal of Tuberculosis and Lung Disease 13, $1320-1330$ (2009).

13. Vilchèze, C., Jacobs, J. \& William, R. The mechanism of isoniazid killing: clarity through the scope of genetics. Annu Rev Microbiol. 61, 35-50 (2007)

14. Vilchèze, C. \& Jacobs, J. R. W. R. Resistance to Isoniazid and Ethionamide in Mycobacterium tuberculosis: Genes, Mutations, and Causalities. Microbiology Spectrum 2, MGM2-0014-2013 (2014).

15. Loessner, M. J. Bacteriophage endolysins-current state of research and applications. Current opinion in microbiology 8, 480-487 (2005).

16. Huang, G. et al. Antibacterial properties of Acinetobacter baumannii phage Abp1 endolysin (PlyAB1). BMC infectious diseases 14, 681 (2014). 
17. Grover, N., Paskaleva, E. E., Mehta, K. K., Dordick, J. S. \& Kane, R. S. Growth inhibition of Mycobacterium smegmatis by mycobacteriophage-derived enzymes. Enzyme and microbial technology 63, 1-6 (2014).

18. Lai, M.-J. et al. Antimycobacterial Activities of Endolysins Derived From a Mycobacteriophage, BTCU-1. Molecules 20, 19277-19290 (2015).

19. Catalão, M. J., Milho, C., Gil, F., Moniz-Pereira, J. \& Pimentel, M. A second endolysin gene is fully embedded in-frame with the lysA gene of mycobacteriophage Ms6. PLoS ONE 6, e20515 (2011).

20. Gil, F. et al. Mycobacteriophage Ms6 LysB specifically targets the outer membrane of Mycobacterium smegmatis. Microbiology 156, 1497-1504 (2010).

21. Pohane, A. A., Joshi, H. \& Jain, V. Molecular Dissection of Phage Endolysin an interdomain interaction confers host specificity in lysin a of mycobacterium phage D29. Journal of Biological Chemistry 289, 12085-12095 (2014).

22. Ronayne, E. et al. P1 Ref Endonuclease: A Molecular Mechanism for Phage-Enhanced Antibiotic Lethality. PLoS genetics 12, e1005797-e1005797 (2016).

23. Fan, X., Teng, T., Wang, H. \& Xie, J. Biology of a novel mycobacteriophage, SWU1, isolated from Chinese soil as revealed by genomic characteristics. Journal of virology 86, 10230-10231 (2012).

24. Fan, X. et al. Genomic and proteomic features of mycobacteriophage SWU1 isolated from China soil. Gene. 561, 45-53 (2015).

25. Pawełczyk, J. \& Kremer, L. The molecular genetics of mycolic acid biosynthesis. Microbiology Spectrum 2, MGM2-0003-2013 (2014).

26. Salzman, V. et al. Transcriptional regulation of lipid homeostasis in mycobacteria. Molecular microbiology 78, 64-77 (2010).

27. Dwyer, D. J. et al. Antibiotics induce redox-related physiological alterations as part of their lethality. Proceedings of the National Academy of Sciences 111, E2100-E2109 (2014).

28. Bass, D. et al. Flow cytometric studies of oxidative product formation by neutrophils: a graded response to membrane stimulation. The Journal of Immunology 130, 1910-1917 (1983).

29. Brandt, R. \& Keston, A. S. Synthesis of diacetyldichlorofluorescin: a stable reagent for fluorometric analysis. Analytical biochemistry 11, 6-9 (1965).

30. Costerton, J. W., Stewart, P. S. \& Greenberg, E. Bacterial biofilms: a common cause of persistent infections. Science 284, 1318-1322 (1999).

31. Yu, J. et al. Both phthiocerol dimycocerosates and phenolic glycolipids are required for virulence of Mycobacterium marinum. Infection and immunity 80, 1381-1389 (2012).

32. Dharmaraja, A. T., Alvala, M., Sriram, D., Yogeeswari, P. \& Chakrapani, H. Design, synthesis and evaluation of small molecule reactive oxygen species generators as selective Mycobacterium tuberculosis inhibitors. Chemical Communications 48, 10325-10327 (2012).

33. Royet, J., Gupta, D. \& Dziarski, R. Peptidoglycan recognition proteins: modulators of the microbiome and inflammation. Nature Reviews Immunology 11, 837-851 (2011).

34. Kashyap, D. R. et al. Peptidoglycan recognition proteins kill bacteria by inducing oxidative, thiol, and metal stress. PLoS Pathogens 10, e1004280 (2014).

35. Fair, R. J. \& Tor, Y. Antibiotics and bacterial resistance in the 21 st century. Perspectives in medicinal chemistry 6, 25 (2014).

36. Kohanski, M. A., Dwyer, D. J., Hayete, B., Lawrence, C. A. \& Collins, J. J. A common mechanism of cellular death induced by bactericidal antibiotics. Cell 130, 797-810 (2007).

37. Koch, A., Mizrahi, V. \& Warner, D. F. The impact of drug resistance on Mycobacterium tuberculosis physiology: what can we learn from rifampicin? Emerging Microbes \& Infections 3, e17 (2014).

38. Kohanski, M. A., Dwyer, D. J. \& Collins, J. J. How antibiotics kill bacteria: from targets to networks. Nature Reviews Microbiology 8, 423-435 (2010).

39. Piccaro, G., Pietraforte, D., Giannoni, F., Mustazzolu, A. \& Fattorini, L. Rifampin Induces Hydroxyl Radical Formation in Mycobacterium tuberculosis. Antimicrobial agents and chemotherapy 58, 7527-7533 (2014).

40. Li, G-q et al. Sublethal vancomycin-induced ROS mediating antibiotic resistance in Staphylococcus aureus. Bioscience reports 35, e00279 (2015).

41. Wu, X., Wang, X., Drlica, K. \& Zhao, X. A toxin-antitoxin module in Bacillus subtilis can both mitigate and amplify effects of lethal stress. PloS ONE 6, e23909-e23909 (2011).

42. Li, L. et al. Ribosomal Elongation Factor 4 Promotes Cell Death Associated with Lethal Stress. mBio. 5, e01708-e01714 (2014).

43. Vandal, O. H. et al. Acid-susceptible mutants of Mycobacterium tuberculosis share hypersusceptibility to cell wall and oxidative stress and to the host environment. Journal of bacteriology 191, 625-631 (2009).

44. Chuang, Y.-M. et al. Deficiency of the Novel Exopolyphosphatase Rv1026/PPX2 Leads to Metabolic Downshift and Altered Cell Wall Permeability in Mycobacterium tuberculosis. mBio. 6, e02428-02414 (2015).

45. Nguyen, H. T., Wolff, K. A., Cartabuke, R. H., Ogwang, S. \& Nguyen, L. A lipoprotein modulates activity of the MtrAB twocomponent system to provide intrinsic multidrug resistance, cytokinetic control and cell wall homeostasis in Mycobacterium. Molecular microbiology 76, 348-364 (2010).

46. Cangelosi, G. A., Palermo, C. O. \& Bermudez, L. E. Phenotypic consequences of red-white colony type variation in Mycobacterium avium. Microbiology 147, 527-533 (2001).

47. Pang, J. M. et al. The polyketide Pks1 contributes to biofilm formation in Mycobacterium tuberculosis. Journal of bacteriology 194, 715-721 (2012).

48. Lee, R., Brennan, P. \& Besra, G. Mycobacterium tuberculosis cell envelope. Tuberculosis. Springer Berlin Heidelberg. 1-27 (1996).

49. Jankute, M., Cox, J. A., Harrison, J. \& Besra, G. S. Assembly of the Mycobacterial Wall. Annual Review of Microbiology 69, 405-423 (2015).

50. Bisson, G. P. et al. Upregulation of the phthiocerol dimycocerosate biosynthetic pathway by rifampin-resistant, rpoB mutant Mycobacterium tuberculosis. Journal of bacteriology 194, 6441-6452 (2012).

51. Xu, W-x. et al. The Wag31 protein interacts with AccA3 and coordinates cell wall lipid permeability and lipophilic drug resistance in Mycobacterium smegmatis. Biochemical and biophysical research communications 448, 255-260 (2014).

52. Rodrigues, L., Ramos, J., Couto, I., Amaral, L. \& Viveiros, M. Ethidium bromide transport across Mycobacterium smegmatis cellwall: correlation with antibiotic resistance. BMC microbiology 11, 35 (2011).

53. Torres-Barceló, C. \& Hochberg, M. E. Evolutionary Rationale for Phages as Complements of Antibiotics. Trends in microbiology 24, 249-256 (2016).

54. Lu, T. K. \& Collins, J. J. Engineered bacteriophage targeting gene networks as adjuvants for antibiotic therapy. Proceedings of the National Academy of Sciences 106, 4629-4634 (2009).

55. Bauer, A., Kirby, W., Sherris, J. C. \& Turck, M. Antibiotic susceptibility testing by a standardized single disk method. American journal of clinical pathology 45, 493 (1966).

56. Mortazavi, A., Williams, B. A., McCue, K., Schaeffer, L. \& Wold, B. Mapping and quantifying mammalian transcriptomes by RNASeq. Nature methods 5, 621-628 (2008).

57. Anders, S., Pyl, P. T. \& Huber, W. HTSeq-A Python framework to work with high-throughput sequencing data. Bioinformatics 31, 166-169 (2014).

58. Robinson, M. D. \& Oshlack, A. A scaling normalization method for differential expression analysis of RNA-seq data. Genome Biol. 11, R25 (2010) 
59. Lewis, T., Nichols, P. D. \& McMeekin, T. A. Evaluation of extraction methods for recovery of fatty acids from lipid-producing microheterotrophs. Journal of Microbiological Methods 43, 107-116 (2000).

60. Luo, H. et al. Mycobacterium tuberculosis Rv1265 promotes mycobacterial intracellular survival and alters cytokine profile of the infected macrophage. Journal of Biomolecular Structure and Dynamics 34, 585-599 (2015).

\section{Acknowledgements}

This work was supported by National Natural Science Foundation [grant numbers 81371851, 81071316, 81271882, 81301394, 81172806, 81471563], New Century Excellent Talents in Universities [grant number NCET11-0703], National Megaprojects for Key Infectious Diseases [grant numbers 2008ZX10003-006, 2008ZX10003001], Excellent Ph.D. thesis fellowship of Southwest University [grant numbers kb2010017, ky2011003], the Fundamental Research Funds for the Central Universities [grant numbers XDJK2014D040, XDJK2016D025], Graduate research and innovation project of graduate in Chongqing (CYS14044), The Chongqing Municipal Committee of Education for postgraduates excellence program [grant numbers YJG123104], and The undergraduates teaching reform program [grant numbers 2013JY201].

\section{Author Contributions}

Q.L., M.Z., X.F. and J.Y. performed the experiments. Q.L. and W.L. analyzed the data. J.X. and W.L. contributed with reagents and materials. Q.L. and J.X. designed the study and wrote the paper.

\section{Additional Information \\ Supplementary information accompanies this paper at http://www.nature.com/srep}

Competing financial interests: The authors declare no competing financial interests.

How to cite this article: Li, Q. et al. Mycobacteriophage SWU1 gp39 can potentiate multiple antibiotics against Mycobacterium via altering the cell wall permeability. Sci. Rep. 6, 28701; doi: 10.1038/srep28701 (2016).

(c) (i) This work is licensed under a Creative Commons Attribution 4.0 International License. The images or other third party material in this article are included in the article's Creative Commons license, unless indicated otherwise in the credit line; if the material is not included under the Creative Commons license, users will need to obtain permission from the license holder to reproduce the material. To view a copy of this license, visit http://creativecommons.org/licenses/by/4.0/ 